\title{
DNA-DNA Recognition: From Tight Contact to Fatal Attraction
}

\author{
Youri Timsit \\ Genomic and Structural Information, \\ CNRS - UPR2589, Institute of Microbiology of the Mediterranean, \\ Aix-Marseille University, \\ Science Park Luminy, Marseille, \\ France
}

\section{Introduction}

The close approach of double helices plays essential roles in the architecture and catalysis of nucleic acids. Transient or long-term DNA-DNA interactions occur in the cell and participate in various genetic functions. For example, bringing DNA sites into proximity is required for DNA recombination, chromatin packaging and building architectural complexes that control transcription and replication (Echols, 1990; Grosschedl, 1995; Segal and Widom, 2009). Thus, the juxtaposition of DNA double helices in a crossover arrangement represents a ubiquitous motif in higher-order DNA structures and is known to be implicated in the genetic functions. The detailed knowledge of the structure and energetics of close DNA-DNA interactions is therefore indispensable for a complete understanding of these functions at the molecular level. Since the backbone of nucleic acids is negatively charged, the close approach of double helices requires cations or polyamines that are present in the cell to reduce their electrostatic repulsion (Bloomfield, 1996). Although similar electrostatic rules govern the assembly of RNA and DNA, the helical packing modes of the two molecular cousins differ as a consequence of their distinct secondary structures. Indeed, in addition to adopt a regular A-conformation, the RNA molecules most often fold into more complex structural motifs due to the presence of the 2'hydroxyl group and extended base pairing rules (Leontis and Westhof, 2001). Thus, RNA structures are characterized by flourishing modes of tertiary interactions, from simple interhelical interactions to the docking of a wide repertoire of sequence-dependent 3D motifs (Leontis et al., 2006; Batey et al., 1999). In contrast, DNA molecules mainly form a regular BDNA double helix stabilized by canonical Watson-Crick base pairing. Consequently, following the evolutionary choice of austerity, the building of higher-order DNA structures is mainly directed by the B-DNA double helix geometry and chirality (Timsit and Moras, 1994).

Although short-range contacts between double helices have been considered to be strongly repulsive, arrays of parallel stacks of helices are formed under various conditions of condensing agents and may form organized phases or DNA liquid crystals (Bloomfield, 1996; Strey et al., 1998). In such arrangements, the inter-axial distance between double 
helical segments is about 25-32 $\AA$ and thus DNA duplexes do not form direct intermolecular interactions (Schellman and Parthasarathy, 1984; Raspaud et al., 2005). In these conditions, the parallel packing of helices is only moderately influenced by the helical nature of DNA (Kornyshev and Leikin, 1998; Minsky, 2004; Kornyshev et al., 2005). However, recent theoretical and experimental studies have indicated that close DNA-DNA interactions can occur in the presence of divalent cations (Qiu et al., 2006; Tan and Chen, 2006; Inoue et al., 2007; Qiu et al., 2007). Indeed, crystallographic studies have shown that both DNA geometry and chirality has a more profound effect on the association of closely-packed DNA helices, as in tight crossover arrangements. Early theoretical studies of helical packing based on simple geometric rules (Srinivasan and Olson, 1992) correctly predicted many of the helical packing modes observed in the crystal structures of nucleic acids (Timsit and Moras, 1992, Murthy and Rose, 2000). This remarkable fit between theoretical and experimental packing without taking into account electrostatic forces therefore indicates that helical interactions in crystals not only minimize the electrostatic repulsion between the negatively charged sugarphosphate backbones but also optimise the docking between the complementary surfaces of the double helices. Indeed, DNA crystals in which electrostatic repulsion between double helices is naturally minimized have unveiled helical packing rules that are dictated by the chirality of the DNA double helix (Timsit and Moras, 1994). Although each type of double helix exposes different accessible surfaces and charged groups that predispose them to interact with themselves in a different manner (Lu et al., 2000), the handedness of A- and Bdouble helices exerts a common constraint on their packing. Thus, the mutual fit of the backbone into the groove specifically generates right-handed crossovers that display particularly interesting structural and functional properties.

\section{From DNA chirality to stable right-handed crossovers}

B-DNA helices can assemble into right-handed DNA crosses by the mutual fit of their sugarphosphate backbone into the major groove (fig. 1a). These right-handed crossovers are characterized by positive values of the crossing angle. The phosphate group penetrates into the major groove to form hydrogen bonds with the amino groups of cytosines (see below) (Timsit et al., 1989). Remarkably, most of the right-handed crosses examined to date are assembled by the major groove-backbone interaction, involve cytosine-phosphate group interaction (fig. 2a) and is stabilized by divalent cations. Although less frequent in DNA crystals, minor groove-backbone interaction has also been observed (Wood et al., 1997). This mode of interaction has also been observed in a molecular dynamics study of mini-circles of DNA (Mitchell et al., 2011). Left-handed B-DNA crossovers that are characterized by negative values $\left(-40\right.$ and $\left.-80^{\circ}\right)$ of the crossing angles prevent the self-fitting of the double helices. Within this geometry, the helices are juxtaposed by groove-groove interactions to minimize their electrostatic repulsion (Timsit et al., 1999) (fig. 1b). However, their mode of interaction is neither stabilized by direct sequence-specific contacts between DNA segments, nor by intermolecular divalent cation bridges.

In contrast, the A-form double helices preferentially self-assemble into right-handed crossovers formed by minor-groove backbone interactions. In A-form double helices, this is the shallow minor groove of the A-form that is devoted to intermolecular interactions. One of the most common elements of the ribosome structure is the interaction of RNA double helices via minor grooves (Nissen et al., 2001). Inter-helical packing involving minor-groove 

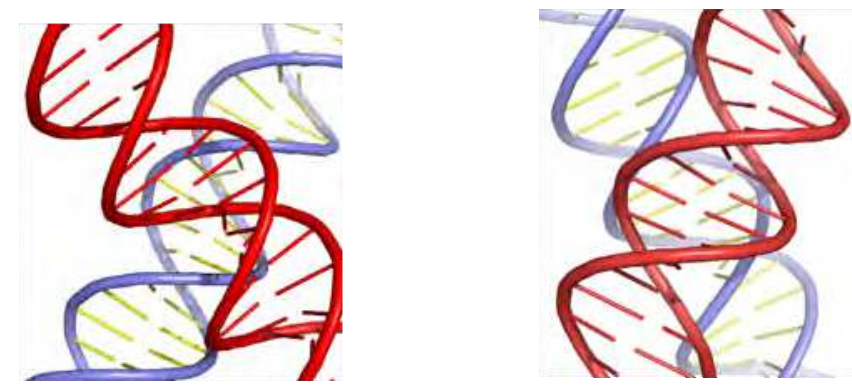

a
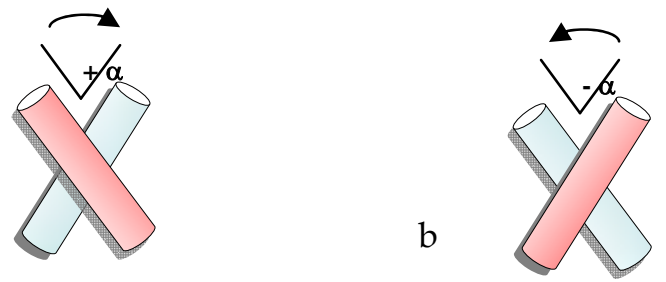

a: Right-handed crossover assembled by the mutual fit of the backbones into the major-groove; b: Lefthanded crossover assembled by major groove-major groove interaction

Fig. 1. Chiral B-DNA crossovers

backbone interactions have been observed in the crystal packing of many RNA oligonucleotides (Schindelin et al., 1995; Baeyens et al., 1995). Moreover, this so called "along-groove" packing motif that has been also observed within the structure of the $23 \mathrm{~S}$ RNA of the large ribosomal subunit is thought to play a role in ribosomal function such as tRNA translocation (Gagnon and Steinberg, 2002; Gagnon et al, 2006). The role of the DNA sequences is also different in the packing of A- and B-DNA helices. Indeed, a comparison of DNA crystal packing modes revealed that the interactions between A-DNA helices are much less dependent from the DNA sequence than the B-DNA ones (Timsit and Moras, 1992). Probably because the shallow minor groove of the A-form provides the opportunity to form many van de Waals and hydrophobic interactions, their stable association has been found less dependent from the formation of specific hydrogen bonds. Thus, a constant geometry of the A-form assemblies can be maintained for a large variety of sequences (Timsit, 1999). In contrast, the tight association of B-DNA helices is greatly influenced by the DNA sequence.

\section{Cytosine and DNA self-assembly}

Right-handed crossovers of B-form DNA double helices are therefore unique in that they are assembled by a sequence-dependent interaction. While the B-DNA double helix dictates the geometry of inter-helical assembly, cytosines play a key role for controlling the interaction through specific interaction of their N4 amino groups with phosphate groups (Timsit et al., 1989; Timsit and Moras, 1991; 1994). Moreover, a recent survey of the Nucleic Acids Database shows that, without exception, cytosine-phosphate interactions are strictly required for stabilising right-handed DNA crossovers (fig. 2a). Probably due to the vicinity 
of the N7 group that displays a negative potential, the N6 amino group of adenine has been not found to substitute the N4 amino group of cytosine for this type of interaction (Timsit and Varnai, 2011).

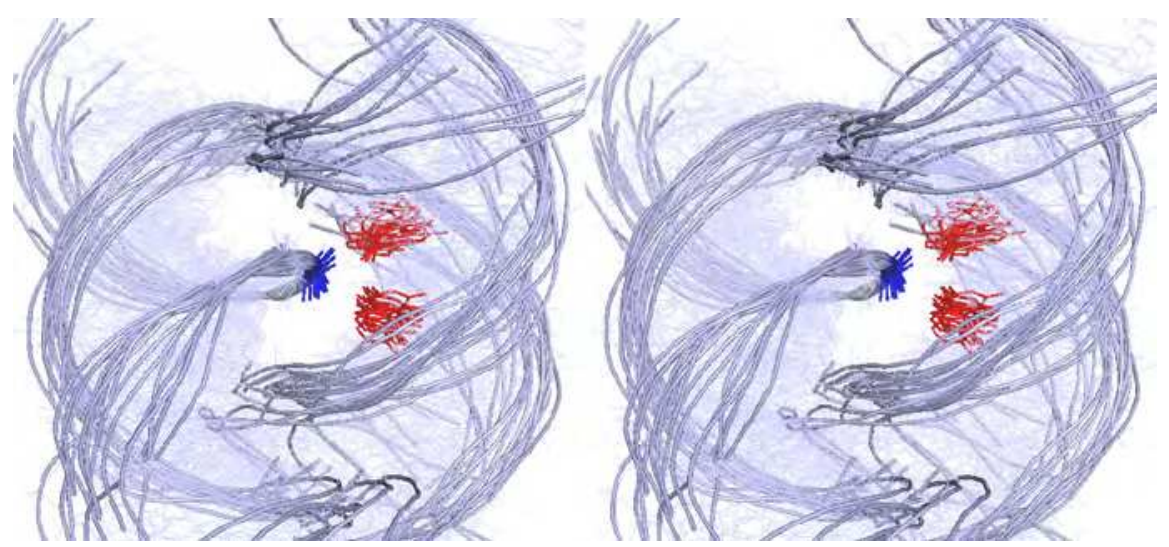

a
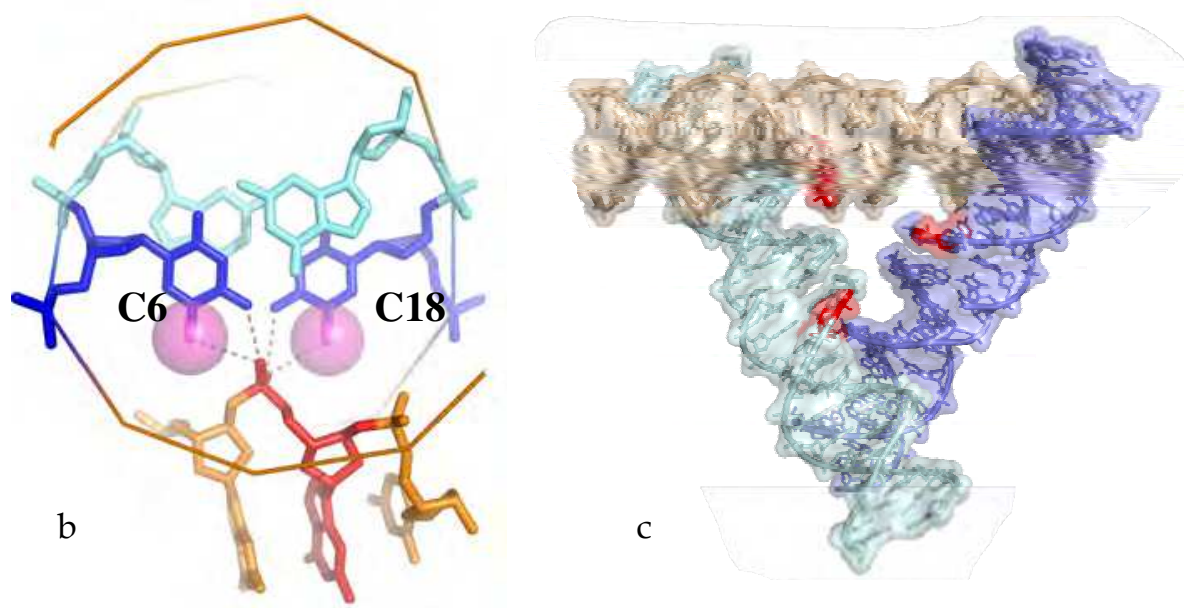

a: Stereo-view of superimposed self-fitted right-handed DNA crossovers found in the Nucleic Acids Database. The cytosines involved in the interaction are represented in red; b: 5-Methyl cytosine stabilizes the groove-backbone interaction through the formation of C-H...O interactions with the phosphate group; c: The role of the sequence in DNA self-assembly: the cytosines (represented in red) dictate the organisation of the triangular motifs

Fig. 2. Cytosine and DNA-DNA recognition

Crystallographic studies of methylated DNA duplexes showed that C5-methyl cytosines also promote and stabilize the formation of DNA crossovers at the modified C5-mpG sequences (Mayer-Jung et al, 1997). The two methyl groups form a hydrophobic clamp which traps the incoming phosphate through $\mathrm{C}-\mathrm{H}$...O interactions that further stabilize the helical assembly (fig. 2b). 
Overall, these works have put in light the particular role of cytosine bases for controlling spatial organisation and the stability of tertiary DNA assemblies. The formations of inter or intramolecular H-bond between the N4 amino group of cytosine and a phosphate group play a key role for controlling DNA-DNA interactions in a sequence dependent manner. These studies provided the basic principles for designing DNA sequence that control the precise organisation of double helices into a 3D lattice. This method for designing DNA crystals has been used successfully for the systematic crystallisation of DNA molecules of various sequences and sizes (Timsit and Moras, 1992; 1996) as for example, the spectacular DNA triangular motifs of DNA dodecamer and decamer duplexes (Timsit et al., 1989; 1991; 1994) (fig. 2c). The DNA triangles recently designed by Zheng et al. (2009) fit remarkably well into this conceptual and methodological framework. Indeed, the overall architecture of these triangles is also dictated by both the cytosine positioning and the double helix geometry (Timsit and Varnai, 2011). The general applicability of these principles has subsequently been supported by the crystal structure of 4-way junctions whose stability also depends on cytosines placed at specific position (Ortiz-Lombardia et al., 1999; Khuu et al., 2006).

\section{Differential stability of chiral crossovers}

The free energy of interactions of DNA duplexes in right and left-handed crossovers as a function of divalent cation concentration in solution has been investigated using molecular dynamics simulations (Varnai and Timsit, 2010). This study showed that right-handed DNA crossovers (fig. 1a) are thermodynamically stable in solution in the presence of divalent cations. Consistent with recent theoretical and experimental observations of close DNADNA interactions in the presence of divalent cations (Qiu et al., 2007; Tan and Chen, 2006; Inoue et al., 2007), a short-range attraction of about $-4 \mathrm{kcal} \mathrm{mol}^{-1}$ between the self-fitted duplexes was observed in the presence of divalent cations (Varnai and Timsit, 2010). Attractive forces at short-range stabilize the DNA-DNA association with inter-axial separation of helices less than $20 \AA$. Right-handed crossovers, however, dissociate in the presence of monovalent ions only. In solution, the acute angle by which the two B-DNA duplexes cross one another in the right-handed geometry fluctuates around an average value of $84 \pm 6^{\circ}$, a value close to that observed in the R3 crystal packing (Timsit et al., 1989). The tight spread around this angle indicates that the major groove induces a strict geometric constraint on the mutually fitted structures. Thus, the crossing angle is mainly influenced by the helical geometry of the B-form duplex that effectively constrains the structure of the assembly by steric interactions in the major groove (Timsit and Moras, 1994). Consistent with the crystallographic studies, molecular dynamics simulation showed that two helices remain assembled by specific cytosine-phosphate interactions and bridging $\mathrm{Mg}^{2+}$ ions at the duplex interface. The repulsion of the negatively charged backbone is circumvented both by the specific relative orientation of helices and by the presence of $\mathrm{Mg}^{2+}$. Therefore, similar structural features of the right-handed crossover are present in solution and in the crystal environment. Simulated DNA triangles constructed from 20-mer sequences are also stabilized by similar interactions in solution. In contrast, left-handed crossovers are unstable at similar ionic conditions and resulted in a swift dissociation of the helices. Without specific intermolecular interaction, left-handed helix juxtapositions by major groove-major groove interaction (fig. $1 \mathrm{~b}$ ) are stable only in the crystallographic environment but appeared to be unstable in solution. 


\section{Role of divalent cations in DNA assembly}

Molecular dynamic simulation studies have also shown that the stabilisation of righthanded crossover increases as the $\mathrm{Mg}^{2+} /$ duplex stoichiometric ratio increases. A minimum of $8 \mathrm{Mg}^{2+}$ per duplex is required to keep the duplexes anchored together with an associated binding free energy of about $-4 \mathrm{kcal} \mathrm{mol}^{-1}$. Higher $\mathrm{Mg}^{2+}$ concentrations $\left(16 \mathrm{Mg}^{2+} /\right.$ duplex $)$ strengthen the helical interaction further and increase the associated binding free energy to $7 \mathrm{kcal} \mathrm{mol}^{-1}$. At lower $\mathrm{Mg}^{2+}$ concentrations $\left(4 \mathrm{Mg}^{2+} /\right.$ duplex) no net attraction was visible. However, monovalent ions cannot replace the effect of $\mathrm{Mg}^{2+}$ to induce attraction between DNA helices even at high $\mathrm{Na}^{+}$concentration (56 Na+/duplex). Importantly, during the simulation, $\mathrm{Mg}^{2+}$ ions occupied the divalent cation binding sites observed in the crystal structure of self-fitted duplexes. In contrast, no specific $\mathrm{Mg}^{2+}$ binding site was observed in the control simulations of isolated duplexes and hence we suggest that specific binding sites are formed simultaneously with the formation of the crossover structure. These data fit well with the crystallographic studies that showed that the diffraction power of crystals of duplexes assembled via groove-backbone interactions was strictly correlated with the $\mathrm{Mg}^{2+}$ /duplex stoichiometric ratio. Best diffracting crystals were obtained with $16 \mathrm{Mg}^{2+}$ per oligonucleotide while very large crystals obtained with $1 \mathrm{Mg}^{2+}$ per oligonucleotide did not diffract at all (Timsit et al., 1989).

The strict requirement for $\mathrm{Mg}^{2+}$ to stabilize tight DNA-DNA interactions is also consistent with recent experimental and theoretical data. For example, SAXS and light scattering experiment indicated DNA-DNA repulsion in the presence of monovalent ions (up to [ $\left.\mathrm{Na}^{+}\right]$ of $600 \mathrm{mM}$ ) but increasing attraction above $\left[\mathrm{Mg}^{2+}\right]$ of $50 \mathrm{mM}$ (Qiu et al., 2007). Also, recent theoretical work that used the tightly bound ion model found that helices repel one other in the presence of monovalent ions while divalent cations are able to induce attraction between two DNA helices (Tan and Chen, 2006). Regions of very tight contacts between DNA segments have been observed in cryo-EM images of supercoiled DNA vitrified from a

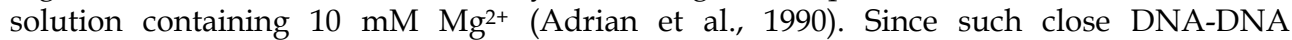
interactions were considered repulsive until recently, these observations were regarded as technical artefacts induced by cryo-congelation. These striking results were, however, supported more recently by AFM studies on supercoiled DNA (Lyubchenko and Shlyakhtenko, 1997; Shlyakhtenko, 2003). In addition, a recent study has also reported that DNA duplexes can self-assemble at nanomolar DNA concentrations in the presence of $\mathrm{Mg}^{2+}$ (Inoue et al., 2007). Divalent cations and in particular $\mathrm{Mg}^{2+}$ ions are also required for the folding of both DNA and RNA molecules. They mediate the folding of Holliday junctions from a planar open structure into a compact stacked conformation (Lilley, 2000). Indeed, four-way junctions and right-handed crosses share an analogous geometry that is stabilized by similar tertiary interactions involving cytosines and $\mathrm{Mg}^{2+}$ (Timsit and Moras, 1991; OrtizLombardia et al., 1999). The folding of particular RNA motifs found in many functional RNA molecules also requires specific divalent cations (van Buuren et al., 2002; Tinoco et al., 1997; Klein et al., 2004; Woodson, 2005). A common feature in most of these structures is the anchoring of a phosphate group to a guanine base through a divalent cation bridge. Thus, among all cations available in physiological conditions, divalent cations have the unique property of stabilising specific and tight intra- and intermolecular interactions between nucleic acid segments by forming guanine-phosphate bridges. In contrast, monovalent ions that are more diffuse around DNA and RNA may have an important role in the long-range steering of duplexes, as for example in the parallel alignment of double helices found in liquid crystals (Strey et al., 1998; Murthy and Rose, 2000). 


\section{DNA sequence and higher-order structures}

These findings revealed that like a directing piece for a "supramolecular construction set", the B-DNA double helix dictates the overall geometry of DNA self-fitted assemblies. Its periodic structure imposes elementary geometric constraints which restrict the spatial organization of DNA segments. They have also demonstrated that the DNA sequence encodes specific signals for positioning intra- or intermolecular DNA-DNA interactions. Cytosine and cluster of guanine bases that constitute preferential divalent cation binding sites act conjointly to define the emplacement of right-handed crossovers. Conversely, AT rich regions are less suitable for tight DNA-DNA interactions. These data may be useful for understanding the organisation of DNA higher-order structures such as, for example, the 30 nm chromatin fibre (Robinson and Rhodes, 2006; Wu et al., 2007). It is well established that electrostatic forces govern primarily the folding of the chromatin fibre (Robinson et al., 2006; Dorigo et al., 2004). The strong dependence of chromatin compaction on cations is reminiscent of a process that involves DNA-DNA interactions. Although recent experimental data support a compact interdigitated solenoidal structure (Robinson et al., 2006), the exact mode of organisation of nucleosomes and linker DNA within the chromatin is still a matter of controversy (Schalch et al., 2005; Dorigo et al., 2004). Consequently, whatever its exact mode of assembly is, chromatin folding is expected to involve close interactions between the linker DNA and/or between the nucleosomal DNA (fig. 3).

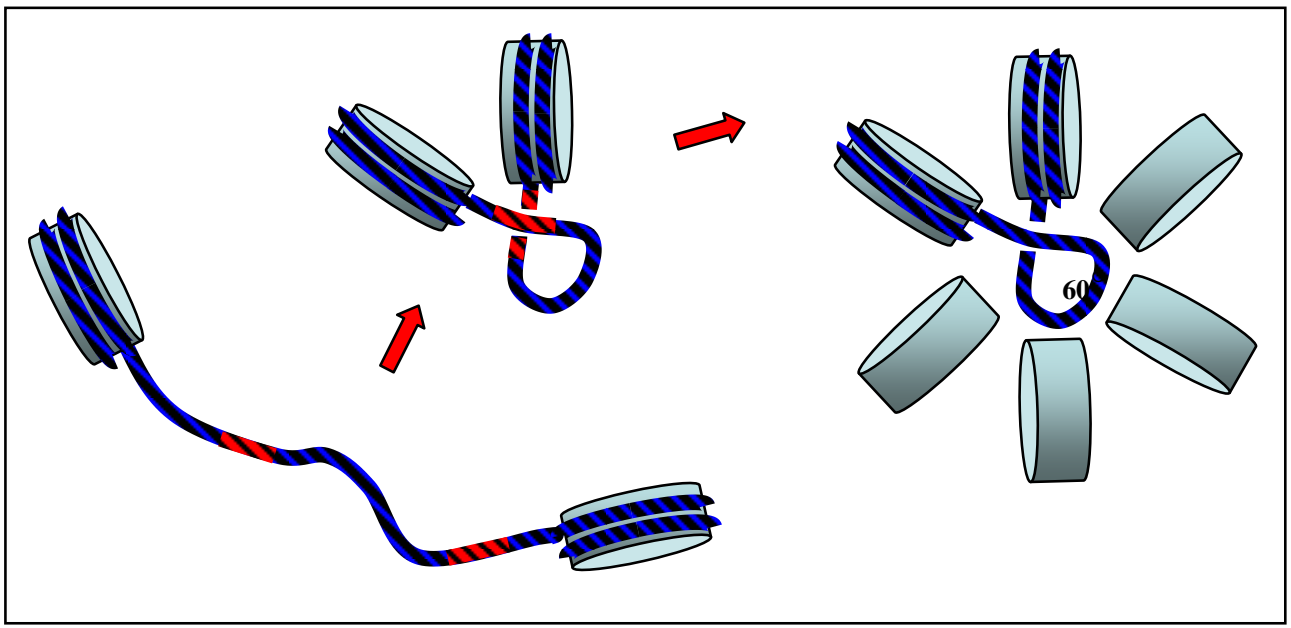

Fig. 3. DNA-DNA recognition may contribute to the assembly of the chromatin fibre.

The GC sequences suitable to form tight DNA-DNA interaction are represented in red. The model proposes that they could contribute to organise tighly packed region in chromatin. The geometric constraints imposed by the groove-backbone interaction may also help to organise the fibre into a symmetric array.

Further, the stability of right-handed crossovers at close to physiological conditions supports earlier hypotheses that groove-backbone fitting organises the nucleosomal or linker assembly within the chromatin fibre (Timsit and Moras, 1991; 1994). Moreover, 
groove-backbone interactions have been observed in the crystal packing of nucleosomes (Davey et al, 2002; Schalch et al., 2005) (fig. 4) and close DNA-DNA interactions are seen in the recent all-atom model of the chromatin fiber (Wong et al., 2007). Therefore, the cell may dispose of a collection of direct DNA-DNA interactions with varying degree of stability that can be exploited for tuning chromatin compaction. In addition, the finding that 5-methyl cytosine promotes tight DNA-DNA interaction may help to understand the role of DNA methylation on the compaction of the chromatin.

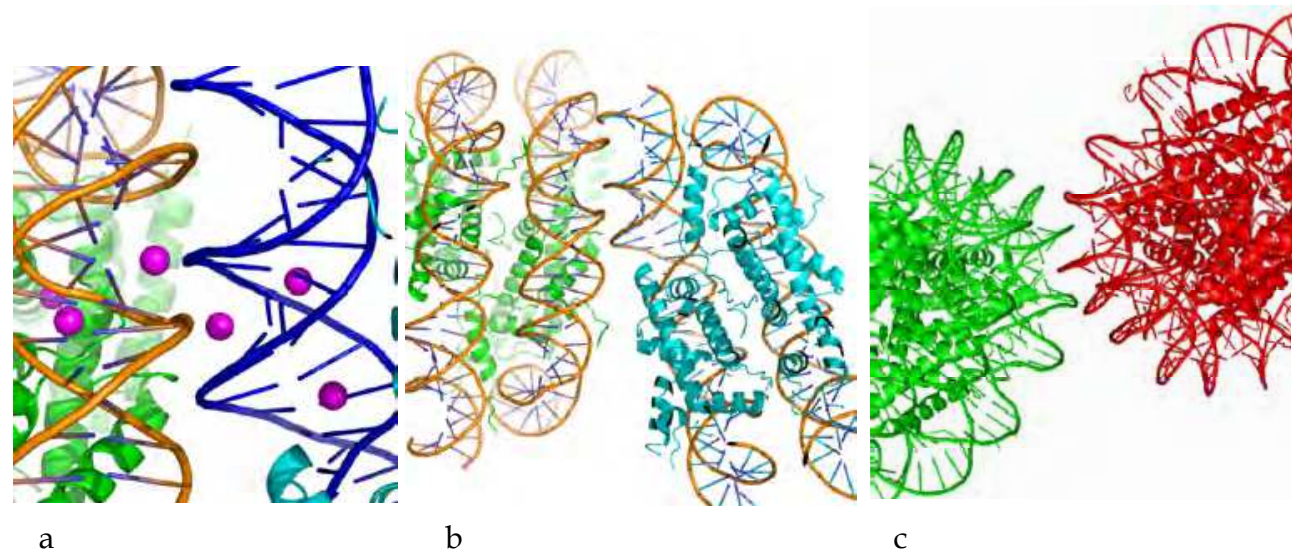

a: Detail of the interaction between two nucleosomes in their crystal packing; the divalent cations that stabilize the interaction are represented in violet sphere (Davey et al., 2002); b: global view of the packing of two nucleosomes intreracting by groove-backbone interaction; c: another mode of self-fitting of two nucleosomes in the crystal structure of Shalch et al. 2005.

Fig. 4. Tight right-handed crossovers between nucleosomal DNA observed in their crystal structure

\section{From crossover geometry to DNA topology}

DNA-DNA interactions have been also found to play a critical role in DNA topology. Indeed, within the interwound plectonemic supercoiled DNA, closely packed regions with intersegmental contacts occur in the presence of divalent cations, under physiological conditions (Vologodskii \& Cozzarelli, 1994; Lyubchenko \& Shlyakhtenko, 1997; Shlyakhtenko et al., 2003). The importance of such close contacts has been also noted for the knotting of supercoiled DNA (Schlick \& Olson, 1992). DNA supercoiling participates in many cellular processes in both prokaryotes and eukaryotes, such as remote gene regulation and site-specific recombination (Wang, 2002; Travers \& Muskhelishvili, 2005). Although DNA is mainly negatively supercoiled in mesophilic cells, transcription and DNA replication may generate domains of positively supercoiled DNA in vivo (Stupina and Wang, 2004; Postow et al., 2001; Liu \& Wang, 1987). Type II topoisomerases can modulate the topological state of DNA by catalyzing the double strand passage reaction. These enzymes not only play a major role in disentangling sister chromatids during replication but also are crucial in maintaining the fine balance of superhelical density and regulating the topological state of genomic DNA (Wang, 2002). 
A remarkable property of type II topoisomerases is their sense of global DNA topology. For example, when a negatively supercoiled ring is singly linked to a nicked ring, these enzymes preferentially unlink the ring rather to remove the supercoils (Roca and Wang, 1996). Topoisomerases II can also simplify DNA topology and reduce the fraction of knotted or catenated circular DNA molecules well below thermodynamic equilibrium values (Rybenkov et al., 1997). They are also capable of chiral discriminiation between knots of opposite sign (Shaw and Wang, 1997) and some of them such as DNA gyrase, topoisomerase IV and human topoisomerase IIa can discern the sign of supercoiled DNA in acting preferentially on positive supercoiled DNA (Crisona et al., 2000, Charvin et al., 2003, Stones et al., 2004; Nöllmann et al., 2007; McClendon et al., 2005). Due to this striking ability to sense the global properties of DNA from local interactions, they have been compared to Maxwell's demons (Pulleyblank, 1997). How topoisomerases can discriminate between the different global topologies of a much larger DNA? Several hypotheses have been postulated for explaining this phenomenon. For example, the protein induces a sharp bend in DNA at the binding site that provides unidirectional strand passage (Rybenkov et al., 2001; Dong and Berger, 2007). A kinetic proofreading model that requires two separate topoisomeraseDNA collisions for segment passage and a model of interaction of the enzyme with three segment-interactions have been also proposed (Yan et al., 1999; Trigueros et al., 2004). Alternatively, it has been suggested that the topological information may be embodied in the local geometry of DNA crossings and that topoisomerases act at the hooked juxtapositions of the strands (Buck and Zechiedrich, 2004; Randall et al., 2006). Indeed, recent experimental studies support this view in showing that topoisomerase IV and DNA gyrase can discriminate the sign of supercoiled DNA on the basis of the geometry of the DNA crossovers (Crisona et al., 2000, Charvin et al., 2003, Stones et al., 2004; Nöllmann et al., 2007; Corbett et al., 2005). Knowing that type II topoisomerases recognise and act on DNA crossovers (Zechiedrich and Osheroff, 1990), the question can be formulated as: how type II topoismerases are able to distinguish the global DNA topology from the local information provided by the juxtaposition of DNA segments?

Applying crystallographic lessons to DNA topology brought useful insights to solve this question. Indeed, the interplay of local and global properties constitutes a key element in the cellular function of DNA and local intra- or intermolecular DNA-DNA interactions play a central role by establishing a link between the two hierarchical levels of structural organisation in DNA (Minsky, 2004). Thus, it has been demonstrated that the various topological states of the cell are associated with different inter-segmental interactions (Timsit and Varnai, 2010). Knowing that right- and left-handed crossovers not only differ by their geometry but also by their stability helped to understand the mechanism of chiral discrimination by type II topoisomerases. Indeed, the stable right-handed DNA crossovers constitute the most probable structure of site juxtaposition in physiological conditions. Consequently, right-handed crosses that occur preferentially in $(+)$ supercoiled DNA for geometrical reasons, is also preferentially formed in the absence of superhelical stress, as in relaxed DNA, catenanes or loose knots for electrostatic reasons. Thus, whereas the unstable left-handed crossovers are exclusively formed in negatively supercoiled DNA, stable righthanded crossovers constitute the local signature of an unusual topological state in the cell, such as the positively supercoiled or relaxed DNA. The differential stability of crossovers may be therefore exploited for sensing the global topology of DNA from local interactions providing a simple mechanism for the local discrimination of global DNA topology. This 
suggested that type II topoisomerases may discriminate (-) supercoiling from other topological states in preferentially acting on stable right-handed crossovers. In addition, a recent study has demonstrated how binding right-handed crossovers across their large angle imposes a different topological link between the type II topoisomerase and the plectonemes of opposite sign. The different topological links affect the enzyme freedom of motion and processivity and provide an explanation for the chiral discrimination (Timsit, 2011).

\section{Right-handed double helix and the evolutionary choice of DNA topology}

The asymmetrical behaviour of supercoiled DNA of opposite signs may have contributed to orient early choices for DNA topology in the nascent DNA world. DNA topology has been the subject of adaptive pressure in organisms that live at different temperatures for maintaining the balance between the melting potential and functional stability (Forterre and Gadelle, 2009). In addition, it is well known that the dynamics of plectonemic DNA supercoiling plays a critical role in promoting interactions between remote sites in processes such as transcription initiation and site-specific recombination (Kanaar and Cozzarelli, 1992; Travers and Muskhelishvili, 2007). Such dynamic should be also finely tuned in function of temperature and the topological state of the supercoiled DNA. Indeed, several studies have shown that some particular local inter-segmental contacts alter the functional dynamic of supercoiled DNA (Minsky, 2004). Similarly, divalent cations that promote formation of stacked 4-way junctions (Lilley, 2000) considerably slow down the kinetics of spontaneous branch migration (Panyutin and Hsieh, 1994).

It is therefore likely that, among other physical properties of DNA, such as its anisotropic flexibility (Olson and Zhurkin, 2000; Travers, 2004), or the fact that DNA is more easily untwisted than overtwisted, the differential stability of chiral crossovers has influenced the choice of DNA topology in mesophilic cells. In particular, the formation of stable righthanded crossovers in relaxed or $(+)$ supercoiled DNA may have posed challenges to mesophilic cells: in the presence of divalent cations, the stable inter-segmental interactions should make (+) supercoiled DNA significantly more "sticky" than (-) supercoiled DNA, along GC rich sequences. Indeed, from a functional point of view, right-handed DNA crosses can be viewed as a Janus-like DNA structure. While the stable inter-segmental interactions can be useful for closely packaging DNA into higher-order DNA structures, they may have a detrimental effect by impeding the global dynamics of the genome, if they occur without control within a plectonemic supercoiled DNA. It is therefore possible that these two opposite features may have lead to different evolutionary strategies to adapt to mesophilic conditions where weak interactions that occur within right-handed crossovers can be expected to be stable.

Indeed, in contrast to life at high temperature that can tolerate various topological states of DNA -from negative supercoiled DNA to slightly positively supercoiled DNA- (BrochierArmanet and Forterre, 2006; Charbonnier and Forterre, 1994; Guipaud et al., 1997; LopezGarcia et al., 2000; Marguet and Forterre, 1994), adaptation to mesophilic life is much more contraining on the topology of DNA: the genome of mesophilic organisms, including bacteria, archaea and eukarya, is systematically (-) supercoiled. All mesophilic bacteria have a DNA gyrase that introduce (-) supercoiling in a plectonemic form (Forterre and Gadelle, 2009). Particularly interesting is the case of mesophilic archaea. They have either acquired a 
gyrase that introduce negative supercoiling, or histones that wrap DNA into toroidal supercoils (Forterre and Gadelle, 2009). In other words, mesophilic organisms appear to have evolved to strictly avoid the presence of permanently relaxed or $(+)$ supercoiled DNA in their genome. As mentioned above these topological states are expected to impede the dynamics of supercoiled DNA and affect functions. Maintaining permanent (-) supercoiling could therefore be viewed as preventing sticky interactions and promoting the "fluidity" required for various functions. Our model can also account for the observation that hyperthermophilic archaea tolerate other topological states of DNA, such as the relaxed or slightly $(+)$ supercoiled states. Indeed, higher temperatures would decrease the stability of right-handed crossovers and restore the relative mobility of DNA segments.

Second, wrapping DNA around histones in mesophilic archaea and eukarya can be viewed as an alternative mode of adaptation to the presence of sticky DNA-DNA interactions in their genome. It can be speculated that this regular mode of DNA packaging allows the organism to precisely control the position of right-handed crosses and to exploit their physical properties. For example, it has been proposed that DNA self-fitting may contribute to stabilise the interactions between nucleosomes or DNA linkers within the chromatin fibre (Robinson and Rhodes, 2006).

\section{Is DNA a helicase?}

In another hand, DNA melting and strand-separation is indispensable for the initiation of the replication and transcription. However, the opening of base pairs in mesophilic conditions in which the double helix is expected to be stable represents another challenging problem. For example, it is commonly thought that the underwound DNA in (-) supercoiled DNA facilitates the strand separation required for transcription or DNA recombination in mesophilic bacteria (Travers and Muskhelishvili, 2007; Benham and Mielke, 2005; Vologodskii and Cozzarelli, 1994). Although it is commonly thought that the less stable AT base pairs are the preferential sites of initiation of DNA melting, experimental data have shown that the question is more complex. For example, it has been shown that the large scale opening of $\mathrm{A}+\mathrm{T}$ rich sequences within supercoiled DNA molecule is suppressed by salts. The authors concluded that the spontaneous opening is highly unlikely under physiological conditions of salt, temperature and superhelicity and that proteins would be required to facilitate opening transitions (Bowater et al., 1994). On the basis of a particular behaviour of specific DNA sequences submitted to tight DNA-DNA interaction, the present study proposes an alternative model for the induction of strand separation in physiological conditions.

Indeed, crystallographic studies have revealed that the groove-backbone interaction can profoundly modify the secondary structure of the double helix in specific sequences (Timsit and Moras, 1995). For example, in the tet d(ACCGGCGCCACA) crystals, the groovebackbone interaction has triggered pre-melting of the double helix (Timsit et al., 1991). This study showed indeed that, in pulling-out the interacting cytosine, the phosphate group inserted into the major groove has broken the corresponding GC base pair in the middle of the duplex. This event has been then propagated on both sides of the anchoring points, to the neighbouring base pairs, thus leading to the concerted shift of the base pairing along the major groove (fig. 5). These observations were correlated with the great thermal sensitivity of the tet crystals and suggested an unstable state of the double helix induced by the tight 
DNA-DNA interactions has been trapped within the crystals at low temperature. Indeed, the disruption of canonical Watson-Crick pairing to form less stable shifted non WatsonCrick pairing is thought to reduce the stability of the double helix.

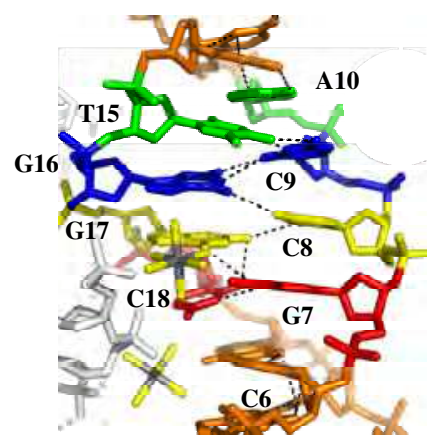

a

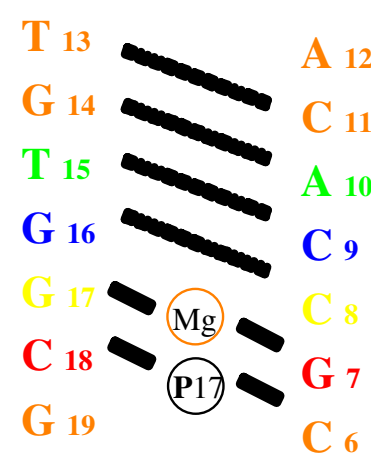

b

a: Shift of the base pairing in the tet dodecamer. The backbone of the fitted molecule is represented in grey. The bases that should be normaly paired are represented with the same color ; $b$ : Schematic view of the shift of the base pairing. The phosphate group inserted into the major groove (P17) has induced the breaking of the G7-C18 base pair

Fig. 5. Base pair opening and helix destabisation induced by DNA-DNA interactions

It has been therefore proposed that this temperature sensitive structure represented an example of "premelted" state induced by DNA-DNA interaction within the crystal (Timsit et al., 1991; Timsit and Moras, 1995). This unique property was found to be related to special features of tet sequence. Indeed, although submitted to identical intermolecular contacts, the structure of the duplex ras d(ACCGCCGGCGCC) remained nearly unaffected and its crystals were stable up to $37^{\circ} \mathrm{C}$. It was therefore concluded that due to its ability to form a rearranged pairing in the major groove and to the presence of highly unstable CA steps, the CCACA 5'-end of tet was responsible of its unusual properties (Timsit and Moras, 1995 ; 1996). This particular electrostatic partition of the strands, consisting of one strand with a set of major groove donor bases (amino groups of A or C) and another one with a set of major groove acceptor bases (carbonyl groups of $\mathrm{G}$ or $\mathrm{T}$ ), is suitable for the propagation in a domino-like motion, of the alterations induced locally by the DNA-DNA intermolecular contacts.

A similar opening of a GC base pair induced by the insertion of phosphate group into the major groove has been also observed in the high resolution structure of a decamer duplex (Van Aalten et al., 1999). However, in that structure, the disruption of the base pairing remained located to the central base-pair and was not propagated since the sequence is not appropriate. In addition, many other examples have shown that the close approach of the helices contributes to destabilise the base pairs (Tarri and Secco, 1995). The analysis of these structures showed that both the duplex sequences and the tightness of the interaction modulate the structural response to DNA-DNA interactions, from the disruption of a single base-pair to the induction of a premelted structure. $(C / A)_{n}$ or $(T / G)_{n}$ sequences were proposed to be particularly sensitive to intermolecular interactions. 


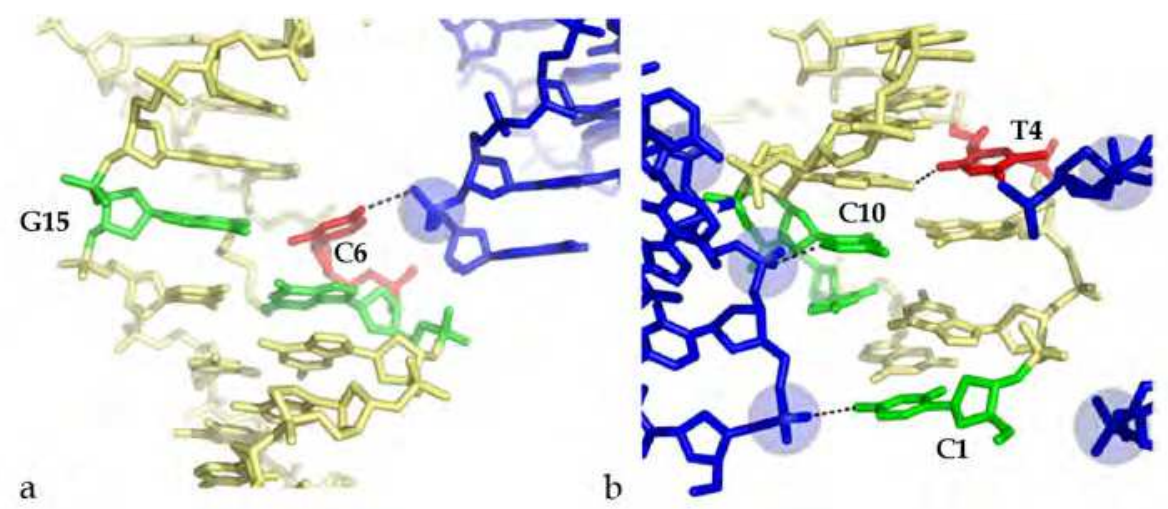

The backbone of the double helix that trigger the disruption of the base pair is represented in blue. a : Van Aalten et al. (1999) ; b : Tari and Secco (1995)

Fig. 6. Inter-helical interaction and base-pair opening

Overall, these findings suggested a molecular mechanism in which the melting of the double helix can be triggered by specific DNA-DNA intermolecular contacts. The close and specific approach of DNA segments occuring in genome packaging, DNA looping, $\left(^{+}\right)$supercoiled DNA or synapsis pairing can trigger, in a sequence-dependent manner, the melting of the double helix and thus initiate the strand separation. In this model, both DNA-DNA

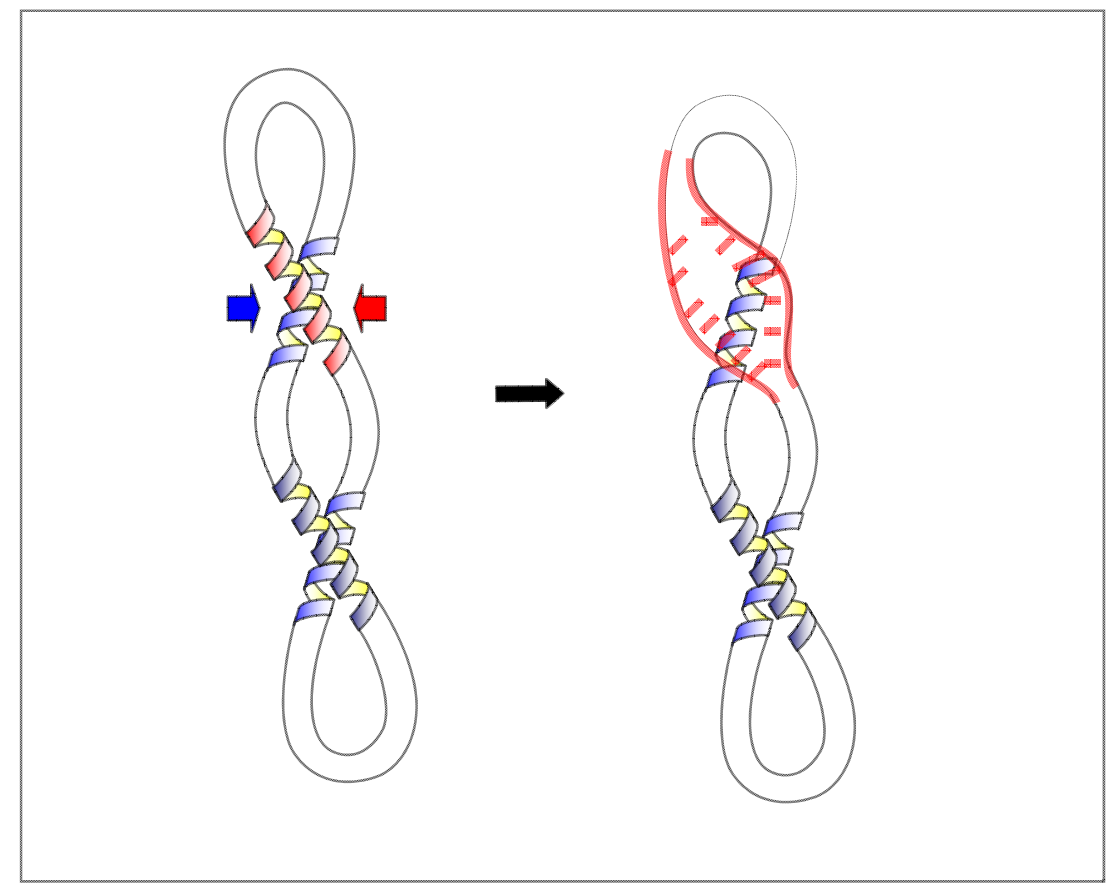

Fig. 7. DNA may be a helicase 
interaction and specific sequences act together as a "catalyst" for triggering and stabilising higher energetic premelted states (transition state) of the DNA. These findings therefore suggest that DNA may realy be a helicase (fig. 7).

The close approach of two DNA segments in supercoiled DNA or higher-order structure can induce the initiation of strand separation in specific sequences such as $(C / A)_{n}$ or $(T / G)_{n}$ represented in red

\section{Conclusion}

This chapter shows firstly, that the double helix geometry and handedness play a critical role in the building rules of DNA higher-order structures. For example, due to the structural and electrostatic complementarity between the grooves and the sugar-phosphate backbone, the double helices can be mutually fitted by major groove-backbone interaction into stable right-handed crossovers. This interaction is specifically stabilised by cytosine bases of one helix that form hydrogen bonds with the phosphate group of another helix. This property has in turn important repercussions on DNA topology and on the mechanism of action of type II topoisomerases. Secondly, on the basis of crystallographic studies, it is shown here that the close DNA-DNA interactions can also trigger strand separation in a specific sequence context, thus raising the provocative question: is DNA a helicase? These observations provide alternative models for understanding mechanism DNA unwinding required in numerous genetic functions such as replication, transcription and recombination.

\section{References}

Adrian, M., ten Heggeler-Bordier, B., Wahli, W., Stasiak, A.Z., Stasiak, A. and Dubochet, J. (1990) Direct visualization of supercoiled DNA molecules in solution. EMBO .J 9, 4551-4554.

Baeyens, K., De Bondt, H.L. and Holbrook, S.R. (1996) Structure of an RNA double helix including uracil-uracil base pairs in an internal loop. Nat. Struct. Biol. 2, 56-62.

Batey, R.T., Rambo, R.P. and Doudna, J.A. (1999) Tertiary motifs in RNA structure and folding. Angew. Chem. Int. Ed. 38, 2326-2343.

Benham, C.J. and Mielke, S.P. (2005) DNA mechanics. Annu. Rev. Biomed. Eng. 7, 21-53.

Bowater, R.P., Aboul-ela, F. and Lilley, D.M. (1994) Large-scale opening of A + T rich regions within supercoiled DNA molecules is suppressed by salt. Nucleic Acids Res. 22, 2042-50.

Brochier-Armanet, C. and Forterre, P. (2006) Widespread archael reverse gyrase in thermophilic bacteria suggest a complex history of vertical inheritance and lateral gene transfers. Archaea 2, 83-93.

Bloomfield, V.A. (1996) DNA condensation. Curr. Opin. Struct. Biol. 6, 334-341.

Buck, G.R. and Zechiedrich, E.L (2004) DNA Disentangling by Type-2 Topoisomerases. J. Mol. Biol. 340, 933-939.

Charbonnier F, Forterre P (1994) Comparison of plasmid DNA topology among mesophilic and thermophilic eubacteria and archaebacteria. J. Bacteriol. 176, 1251-1259. 
Charvin, G., Bensimon, D. and Croquette, V. (2003) Single-molecule study of DNA unlinking by eukaryotic and prokaryotic type-II topoisomerases. Proc. Natl. Acad. Sci. USA 100, 9820-9825.

Corbett, K.D, Schoeffler, A.J, Thomsen, N.D. and Berger, J.B. (2005) The structural basis for substrate specificity in DNA topoisomerase IV. J. Mol. Biol. 351, 545-561.

Crisona, N.J., Strick, T.R., Bensimon, D., Croquette, V. and Cozzarelli, N.R. (2000) Preferential relaxation of positively supercoiled DNA by E. coli topoisomerase IV in single-molecule and ensemble measurements. Gene \& Dev. 14, 2881-2892.

Dong, K.C. and Berger, J.M. (2007) Structural basis for gate-DNA recognition and bending by type IIA topoisomerases. Nature 450, 1201-1206.

Echols, H. (1990) Nucleoprotein Structures Initiating DNA-Replication, Transcription, and Site-Specific Recombination. J. Biol. Chem. 265, 14697-14700.

Forterre, P. and Gadelle, D. (2009) Phylogenomics of DNA topoisomerases: their origin and putative roles in the emergence of modern organisms. Nucleic Acids Res. 37, 679-692.

Guipaud, O., Marguet, E., Noll, K., Bouthier de la Tour, C. and Forterre, P. (1997) Both gyrase and reverse gyrase are present in the hyperthermophilic bacterium Thermogata maritima. Proc. Natl. Acad. Sci. USA 94, 10606-10611.

Grosschedl, R. (1995) Higher-Order Nucleoprotein Complexes in Transcription - Analogies with Site-Specific Recombination. Curr. Opin. Cell Biol. 7, 362-370.

Inoue, S., Sugiyama, S., Travers, A.A. and Ohyama, T. (2007) Self-assembly of doublestranded DNA molecules at nanomolar concentrations. Biochemistry 46, 164-171.

Khuu, P., Regier Voth, A., Hays, F. and Ho, P.S. The stacked-X DNA Holliday junction and protein recognition J. Mol. Recogn. 19, 234-242 (2006).

Klein, D.J., Moore, P.B. and Steitz, T.A. (2004) The contribution of metal ions to the structural stability of the large ribosomal subunit. RNA 10, 1366-1379.

Kornyshev, A.A. and Leikin, S. (1998) Electrostatic interaction between helical macromolecules in dense aggregates: an impetus for DNA poly- and mesomorphism. Proc. Natl. Acad. Sci. USA 95, 13579-13584.

Kornyshev, A.A., Lee, D.J., Leikin, S., Wynveen, A. and Zimmerman, S.B. (2005) Direct observation of azimuthal correlations between DNA in hydrated aggregates. Phys. Rev. Lett. 95, 2537-2540.

Leontis, N.B. and Westhof, E. (2001). Geometric nomenclature and classification of RNA base pairs. RNA 7, 499-512.

Leontis, N.B., Lescoute, A., and Westhof, E. (2006). The buidling blocks and motifs of RNA architecture. Curr. Opin. Struct. Biol. 16, 279-287.

Lilley, D.M.J. (2000) Structures of helical junctions in nucleic acids. Q. Rev. Biophys. 33, 109159.

Liu, L.F. and Wang, J.C. (1987) Supercoiling of the DNA template during transcription. Proc. Natl. Acad. Sci. USA. 84, 7024-7027.

Liu, Z., Deibler, R.W., Chan, H.S. and Zechiedrich, L. (2009) The why and how DNA unlinking. Nucl. Acid Res. 37 661-671.

Liu, Z., Mann, J.K., Zechiedrich, E.L. and Chan, H.S. (2006) Topological information embodied in local juxtaposition geometry provides a statistical mechanical basis for unknotting by type-2 DNA topoisomerases. J. Mol. Biol. 361, 268-285.

Lopez-Garcia, P., Forterre, P., Van der Oost, J. and Erauso, G. (2000) Plasmid pGS5 from hyperthermophilic archaeon archaeoglobus profundus is negatively supercoiled. $J$. Bacteriol. 182, 4998-5000. 
Lu, X.-J., Shakked, Z. and Olson, W. (2000). A-form conformational motifs in ligand-bound DNA structures. J. Mol. Biol. 300, 819-840.

Lyubchenko, Y.L. and Shlyakhtenko, L.S. (1997) Visualization of supercoiled DNA with atomic force microscopy in situ. Proc. Natl. Acad. Sci. USA 94, 496-501.

Marguet, E. and Forterre, P. (1994) DNA stability at temperatures typical for hyperthermophiles Nucleic Acids Res. 22, 1681-1686.

Mayer-Jung, C., Moras, D. and Timsit, Y. (1997). Effect of cytosine methylation on DNADNA recognition at CpG steps J. Mol. Biol. 270, 328-335.

McClendon, A.K., Rodriguez, A.C. and Osheroff, N. (2005) Human topoisomerase IIa rapidly relaxes positively supercoiled DNA. Implications for enzyme action ahead of replication forks. J. Biol. Chem. 280, 39337-39345.

Minsky, A. (2004) Information content and complexity in the high-order organization of DNA. Annu. Rev. Biophys. Biomol. Struct. 33, 317-342.

Mitchell, J.S., Laughton, C.A. and Harris, S.A. Atomistic simulations reveal bubbles, kinks and wrinkles in supercoiled DNA. Nucl. Acids Res., 1-11 doi:10.1093/nar/gkq1312

Murthy, V.L. and Rose, G.D. (2000) Is counterion delocalization responsible for collapse in RNA folding? Biochemistry 39, 14365-14370.

Nissen, P., Ippolito, J.A., Ban, N., Moore, P. and Steitz, T. (2001) RNA tertiary interactions in the large ribosomal subunit: the A-minor motif. Proc. Natl. Acad. Sci. USA 98, 48994903

Nöllmann, N., Stone, M.D., Bryant, Z., Gore, J., Crisona, N.J., Hong, S.C., Mitelheiser, S., Maxwell, A., Bustamante, C. and Cozzarelli, N.R. (2007) Multiple modes of Escherichia coli DNA gyrase activity revealed by force and torque. Nat. Struct. Mol. Biol. 14, 264-271.

Olson, W.K., and Zhurkin, V.B. (2000) Modeling DNA deformations. Curr.Opin. Struc. Biol. 10, 286-297.

Ortiz-Lombardia, M., Gonzalez, A., Eritja, R., Aymami, J., Azorin, F. and Coll, M. Crystal structure of a DNA Holliday junction. Nat. Struct. Biol. 6, 913-917 (1999).

Postow, L., Crisona, N.J., Peter, B.J., Hardy, C.D. and Cozzarelli, N.R. (2001) Topological challenges to DNA replication: conformations at the fork. Proc. Natl. Acad. Sci. USA 98, 8219-8226.

Pulleyblank, D.E. (1997) Of Topo and Maxwell's dream. Science 277, 648-649.

Qiu, X.Y., Kwok, L.W., Park, H.Y., Lamb, J.S., Andresen, K. and Pollack, L. (2006) Measuring inter-DNA potentials in solution. Phys. Rev. Lett. 96, 138101-138104.

Qiu, X., Andresen, K., Kwok, L.W., Lamb, J.S., Park, H.Y. and Pollack, L. (2007) Inter-DNA attraction mediated by divalent counterions. Phys. Rev. Lett. 99, 038104-038107.

Randall, G.L., Pettitt, B.M., Buck, G.R. and Zechiedriech, E.L. (2006) Electrostatics of DNADNA juxtapositions: consequences for type II topoisomerase function. J. Phys. Condens. Matter 18, S173-S185.

Raspaud, E., Durand, D. and Livolant, F. (2005) Interhelical spacing in liquid crystalline spermine and spermidine-DNA precipitates. Biophys. J. 88, 392-403.

Roca, J. and Wang, J.C. (1996) The probabilities of supercoil removal and decatenation by yeast DNA topoisomerase II. Genes Cells 1, 17-27.

Robinson, P.J.J. and Rhodes, D. (2006) Structure of the ' $30 \mathrm{~nm}$ ' chromatin fibre: A key role for the linker histone. Curr. Opin. Struct. Biol. 16, 336-343.

Rybenkov, V.V., Ullsperger, C., Vologodskii, A.V. and Cozzarelli, N.R. (1997) Simplification of DNA topology below equilibrium values by type II topoisomerases. Science 277, 690-693. 
Schalch, T., Duda, S., Sargent, D.F. and Richmond, T.J. (2005) X-ray structure of a tetranucleosome and its implications for the chromatin fibre. Nature 436, 138-141.

Schellman, J.A. and Parthasarathy, N. (1984) X-Ray-diffraction studies on cation-collapsed DNA. J. Mol. Biol., 175, 313-329.

Shaw, S. and Wang, J.C. (1997) Chirality of DNA trefoils: implications in intramolecular synapsis of distant DNA segments. Proc. Natl. Acad. Sci. USA 94, 1692-1697.

Segal, E. and Widom, J. (2009) What controls nucleosome positions? Trends Genet. 25, 335343.

Schlick, T. and Olson, W.K. (1992) Trefoil Knotting Revealed by Molecular-Dynamics Simulations of Supercoiled DNA. Science 257, 1110-1115.

Schindelin, H., Zhang, M., Bald, R., Fürste, J.-P., Erdmann, V.A. and Heinemann, U. (1995) Crystal structure of an RNA dodecamer containing the Escherichia coli ShineDalgarno sequence. J. Mol. Biol. 249, 595-603.

Shlyakhtenko, L.S., Miloseska, L., Potaman, V.N., Sinden, R.R. and Lyubchenko, Y.L. (2003) Intersegmental interactions in supercoiled DNA: atomic force microscope study. Ultramicroscopy 97, 263-270.

Stone, M.D., Bryant, Z., Crisona, N.J., Smith, S.B., Vologodskii, A., Bustamente, C. and Cozzarelli, N.R. (2003) Chirality sensing by Escherichia coli topoisomerase IV and the mechanism of type II topoisomerases. Proc. Natl. Acad. Sci. USA 100, 8654-8659.

Strey, H.H., Podgornik, R., Rau, D.C. and Parsegian, V.A. (1998) DNA--DNA interactions. Curr. Opin. Struct. Biol. 8, 309-313.

Stupina, V.A and Wang, J.C. (2004) DNA axial rotation and the merge of oppositely supercoiled DNA domains in Escherichia coli: effects of DNA bends. Proc. Natl. Acad. Sci. USA 101, 8608-8613.

Tan, Z.J. and Chen, S.J. (2006) Electrostatic free energy landscapes for nucleic acid helix assembly. Nucleic Acids Res. 34, 6629-6639.

Tari, L.W. and Secco, A.S. (1995) Base-pair opening and spermine binding--B-DNA features displayed in the crystal structure of a gal operon fragment: implications for protein-DNA recognition. Nucleic Acids Res. 23, 2065-2073.

Timsit, Y., Westhof, E., Fuchs, R and Moras. D. Unusual helical packing in crystals of DNA bearing a mutation hot spot. Nature 341, 459-462 (1989).

Timsit, Y., Vilbois, E. and Moras, D. Base pairing shift in the major groove of $(\mathrm{CA})_{\mathrm{n}}$ tracts by B-DNA crystal structures. Nature 354, 167-170 (1991).

Timsit, Y. and Moras, D. (1991) Groove-Backbone Interaction in B-DNA - Implication for DNA condensation and recombination. J. Mol. Biol. 221, 919-940.

Timsit, Y. and Moras, D. (1992) Crystallization of DNA. Meth. in Enzymology 211, 409-429.

Timsit, Y. and Moras, D. (1994) DNA self-fitting: the double helix directs the geometry of its supramolecular assemblies. EMBO J. 13, 2737-2746.

Timsit, Y. and Moras, D. (1995). Self-fitting and self-modifying properties of the B-DNA molecule. J.Mol. Biol. 251, 629-647.

Timsit Y and Moras D. (1996). Cruciform structures and functions. Q. Rev. Biophys. 29, 279307.

Timsit, Y., Shatzky-Schwartz, M. and Shakked, Z. (1999) Left-handed DNA crossovers. Implications for DNA-DNA recognition and structural alterations. J. Biomol. Struct. Dyn. 16, 775-785.

Timsit, Y. (1999) DNA structure and polymerase fidelity. J. Mol. Biol. 293, 835-853. 
Timsit, Y. and Varnai P. (2010) Helical chirality: a link between local interactions and global topology in DNA. Plos One 5:e9326.

Timsit, Y. and Varnai, P. (2011) Cytosine, the double helix and DNA self-assembly. J. Mol. Recognit. 24, 137-138.

Timsit, Y. (2011) Local sensing of global DNA topology: from crossover geometry to type II topoisomerase processivity. Nucleic Acids Res. doi: 10.1093/nar/gkr556 First published online: July 15, 2011

Tinoco, I., Jr. and Kieft, J.S. (1997) The ion core in RNA folding. Nat. Struct. Biol. 4, 509-512.

Travers, A., Muskhelishvili, G. (2007) A common topology for bacterial and eukaryotic transcription initiation? EMBO Rep. 8, 147-151.

Travers, A.A. (2004) The structural basis of DNA flexibility. Philos. T. Roy. Soc. A 362, 14231438.

Trigueros, S., Salceda, J., Bermudez, I., Fernandez, X. and Roca, J. (2004) Asymmetric removal of supercoils suggests how topoisomerase II simplifies DNA topology. J. Mol. Biol. 335, 723-731.

van Aalten D.M., Erlanson D.A., Verdine G.L., Joshua-Tor L. (1999) A structural snapshot of base-pair opening in DNA. Proc Natl Acad Sci USA. 96, 11809-11814.

van Buuren, B.N.M., Hermann, T., Wijmenga, S.S. and Westhof, E. (2002) Browniandynamics simulations of metal-ion binding to four-way junctions. Nucleic Acids Res. 30, 507-514.

Varnai, P. and Timsit, Y. (2010) Differential stability of chiral DNA crossovers mediated by divalent cations. Nucl. Acid Res., 38 4163-4172.

Vologodskii AV, Cozzarelli NR (1994) Conformational and thermodynamic properties of supercoiled DNA. Annu Rev Biophys Biomol Struct 23: 609-643.

Vologodskii, A. (2009) Theoretical models of topology simplification by type IIA DNA topoisomerases. Nucl. Acid Res. 37, 3125-3133.

Wang, J.C. (2002) Cellular roles of DNA topoisomerases: a molecular perspective. Nature Rev., 3, 430-440.

Wong, H., Victor, J.M. and Mozziconacci, J. (2007) An all-atom model of the chromatin fiber containing linker histones reveals a versatile structure tuned by the nucleosomal repeat length. PloS one, 2, e877.

Wood, A.A., Nunn, C.M., Trent, J.O. and Neidle, S. (1997) Sequence-dependent crossed helix packing in the crystal structure of a B-DNA decamer yields a detailed model for the Holliday junction. J. Mol. Biol. 269, 827-841.

Woodson, S.A. (2005) Metal ions and RNA folding: a highly charged topic with a dynamic future. Curr. Opin. Chem. Biol., 9, 104-109.

$\mathrm{Wu}$, C.Y., Bassett, A. and Travers, A. (2007) A variable topology for the 30-nm chromatin fibre. Embo Rep. 8, 1129-1134.

Yan, J., Magnasco, M.O. and Marko J.F. (1999) A kinetic proofreading mechanism for disentanglement of DNA by topoisomerases. Nature 401, 932-935.

Zechiedrich, E.L. and Osheroff, N. (1990) Eukaryotic topoisomerases recognize nucleic acid topology by preferentially interacting with DNA crossovers. EMBO J. 9, 4555-4562.

Zheng, J., Birktoft, J.J., Chen, Y., Wang, T., Sha, R., Constantinou, P.E., Ginell, S.L., Mao, C., \& Seeman, N.C. From molecular to macroscopic via the rational design of selfassembled 3D DNA crystal. Nature 461, 74-77 (2009). 


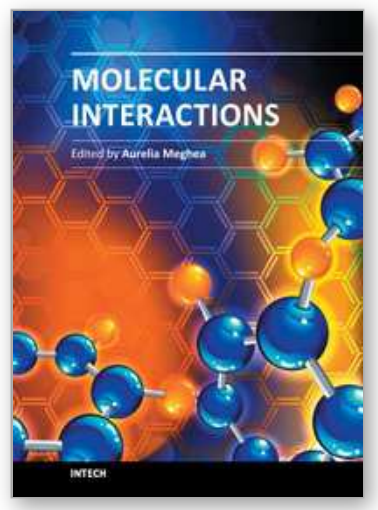

\author{
Molecular Interactions \\ Edited by Prof. Aurelia Meghea
}

ISBN 978-953-51-0079-9

Hard cover, 400 pages

Publisher InTech

Published online 29, February, 2012

Published in print edition February, 2012

In a classical approach materials science is mainly dealing with interatomic interactions within molecules, without paying much interest on weak intermolecular interactions. However, the variety of structures actually is the result of weak ordering because of noncovalent interactions. Indeed, for self-assembly to be possible in soft materials, it is evident that forces between molecules must be much weaker than covalent bonds between the atoms of a molecule. The weak intermolecular interactions responsible for molecular ordering in soft materials include hydrogen bonds, coordination bonds in ligands and complexes, ionic and dipolar interactions, van der Waals forces, and hydrophobic interactions. Recent evolutions in nanosciences and nanotechnologies provide strong arguments to support the opportunity and importance of the topics approached in this book, the fundamental and applicative aspects related to molecular interactions being of large interest in both research and innovative environments. We expect this book to have a strong impact at various education and research training levels, for young and experienced researchers from both academia and industry.

\title{
How to reference
}

In order to correctly reference this scholarly work, feel free to copy and paste the following:

Youri Timsit (2012). DNA-DNA Recognition: From Tight Contact to Fatal Attraction, Molecular Interactions, Prof. Aurelia Meghea (Ed.), ISBN: 978-953-51-0079-9, InTech, Available from:

http://www.intechopen.com/books/molecular-interactions/dna-dna-recognition-from-tight-contact-to-fatalattraction

\section{INTECH}

open science | open minds

\section{InTech Europe}

University Campus STeP Ri

Slavka Krautzeka 83/A

51000 Rijeka, Croatia

Phone: +385 (51) 770447

Fax: +385 (51) 686166

www.intechopen.com

\section{InTech China}

Unit 405, Office Block, Hotel Equatorial Shanghai

No.65, Yan An Road (West), Shanghai, 200040, China

中国上海市延安西路65号上海国际贵都大饭店办公楼405单元

Phone: +86-21-62489820

Fax: +86-21-62489821 
(C) 2012 The Author(s). Licensee IntechOpen. This is an open access article distributed under the terms of the Creative Commons Attribution 3.0 License, which permits unrestricted use, distribution, and reproduction in any medium, provided the original work is properly cited. 